\title{
Foreword to the Special Issue Years in Biophotonics: 70th Anniversary of Prof. A.V. Priezzhev
}

We are pleased to present the special issue of JBPE dedicated to the celebration of the 70th anniversary of Prof. Alexander V. Priezzhev and aimed to honor his contribution to the field of biophotonics.

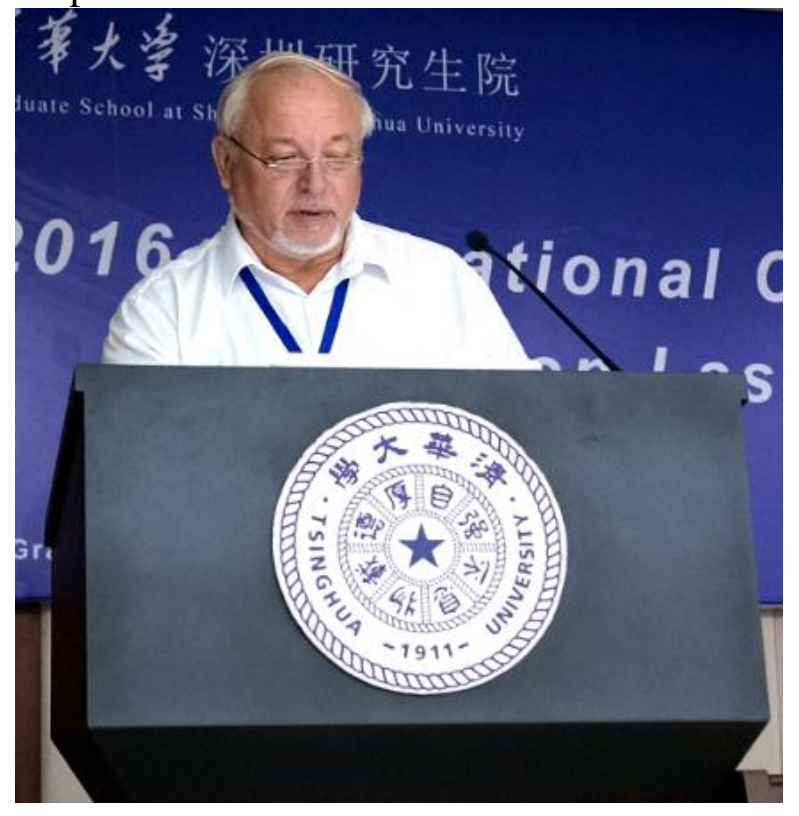

Alexander V. Priezzhev was born on April, 3, 1947. He graduated and received his $\mathrm{PhD}$ degree from the Department of Physics, M.V. Lomonosov Moscow State University (Moscow, Russia) in 1971 and 1975, respectively. Currently he is the head of the Laboratory of Laser Biomedical Photonics at M.V. Lomonosov Moscow State University. As a principal investigator or research fellow he was involved in numerous national and international research projects in the area of medical physics and biomedical optics; he co-authored more than 350 peer-reviewed papers, books and conference proceedings. Alexander V. Priezzhev has been awarded by the Ministry of Healthcare of Russian Federation for long lasting activities in development of novel optical techniques for medical applications. $\mathrm{He}$ also received the award by the Ministry of Science and Education of Russian Federation for achievements in research and education activities. His areas of expertise cover biophysics, biophotonics, biomedical optics, laser medicine, medical physics, physics of biological fluids, nanobiophotonics, and optical and laser measurement techniques.

This issue presents a collection of state-of-the-art research papers in the field of biophotonics closely related to the scientific interests of Prof. A.V. Priezzhev

The paper by Anna N. Yaroslavsky and co-authors from University of Massachusetts in Lowell and Massachusetts General Hospital (USA) reports on multimodal terahertz-optical imaging for delineating nonmelanoma skin cancers providing high increase in sensitivity and specificity of cancer detection and demonstrating great potential for intraoperative assessment of tumor margins.

Alexander Krasnovsky and Anton Kozlov from A.N. Bach Institute of Biochemistry RAS and M.V. Lomonosov Moscow State University (Russia) discuss photooxygenation rates of singlet oxygen traps upon direct laser excitation of oxygen in air-saturated organic solvents and water. The results are important for both analyses of oxygen photonics and dosimetry of laser radiation in biomedical experiments.

Diffuse optical spectroscopy is one of the perspective diagnostics techniques for a variety of biomedical applications connected with monitoring of tissue oxygenation. The Monte-Carlo simulation of signal formation in such system is performed by Daria A. Loginova with co-authors from the Institute of Applied Physics RAS and N. I. Lobachevsky State University of Nizhny Novgorod (Russia). The main aim of this paper is to evaluate the probing depth in different probing configuration that cannot be measured directly, however, it is very important for the optimal technique performance. The authors study different illumination patterns and their efficiency for diffuse optical spectroscopy applications.

Investigation of different algorithms of tissue structural analysis based on optical coherence tomography is presented in paper of Dmitry S. Raupov with co-authors from Samara National Research University (Russia). The authors propose anisotropic diffusion speckle noise reduction 
pre-processing and multimodal texture approach for identification of skin cancer in optical coherence tomography images, involving Haralick texture features, fractal dimension, complex directional field features and Markov random field method. High efficiency of multimodal texture analysis in differentiation between tumor and healthy tissue is demonstrated.

Yu-Chung Lin with co-authors from National Dong Hwa University (Taiwan), Institute of Physics (Taiwan) and P. N. Lebedev Physics Institute RAS (Russia) reports on the perspectives of functionalized nanodiamond complexes in biomedical applications. In particular, nanodiamondberberine complexes are demonstrated to be an efficient tool where nanodiamonds serve as a drug carrier for target delivery while berberine acts as an antimicrobial drug. Fluorescence imaging techniques are employed for delivery monitoring.

Elina Genina with co-authors from Saratov National Research State University, Institute of Precision Mechanics and Control RAS and National Research Tomsk State University (Russia) reports specific features of the optical clearing of the human dura mater in vitro under the action of glucose solutions. The relative diffusion coefficients of glucose are calculated based on the analysis of the collimated transmittance kinetics. The possibility of efficient reduction of light scattering is demonstrated.

Sergey N. Savenkov with co-authors from T. Shevchenko National University of Kyiv (Ukraine), M.V. Lomonosov Moscow State University (Russia), and University of Eastern Finland (Finland) reports on application of optical diagnostics for material characterization. The authors employed Mueller matrix polarimetry to study the samples of microcrystalline cellulose with different porosity. It was demonstrated that this approach provides high accuracy in non-destructive porosity measurements.

Inga Saknite with co-authors from University of Latvia and Riga Stradins University (Latvia) report on application of optical diagnostics for monitoring short-term and long-term effect of novel moisturizing cream on human skin. Combined employment of optical spectroscopy and skin impedance measurements allowed them to monitor skin hydration at different measurement depths.

Herbert Schneckenburger and co-authors from Aalen University (Germany) demonstrate high potential of modern microscopy modalities for biomedical studies at cellular level. Different focus positions with respect to the sample are discussed. The considered microscopy modalities include light scattering microscopy with angular resolution and structured illumination microscopy.

Olga Baum and co-authors from Institute of Photonic Technologies (Russia), B.I. Stepanov Institute of Physics (Belarus), State Research Institute of Eye Diseases (Russia), and M.V. Lomonosov Moscow State University (Russia) presents the concept of the laser activation in drainage system function of eye sclera for two anatomic channels of eye fluid outflow. The study combines experiment with extensive theoretical modelling that allowed to calculate thermal stresses within eye tissues and predict development of cavitation processes in eye sclera that is key for optimizing modes of laser glaucoma treatment.

Leonid Bass with co-authors from Institute of Applied Mathematics RAS (Russia), University of Oulu (Finland) and Institute of Applied Physics RAS (Russia) reports on finite difference approach to solution of the radiative transfer equation (RTE) in general form both for stationary and nonstationary cases. Obtained solutions are compared with the results of Monte Carlo simulations and solution of RTE in diffusion approximation. Applications of the finite difference approach to blood sensing and vessel imaging are reviewed.

In overall, the papers collected in this special issue demonstrate well the exciting potential of biophotonics for biomedical studies and applications, their wide spread throughout the world.

On behalf of all authors, we would like to congratulate Alexander Vasilyevich on his $70^{\text {th }}$ birthday, and wish him many health, many new ideas and discoveries, and successes in all kinds of activities! 
Special issue Editors:

Mikhail Yu. Kirillin, PhD, DSc (Tech)

Laboratory of Biophotonics,

Institute of Applied Physics RAS,

Nizhny Novgorod, Russia

\section{Juergen Lademann, Prof. Dr. Dr.-Ing.}

Center of Experimental and Applied Cutaneous Physiology, Department of Dermatology, Venerology and Allergology, Charité - Universitätsmedizin Berlin, Germany

\section{Valery P. Zakharov, PhD, DSc}

Department of Laser and Biotechnical Systems, Samara National Research University,

Samara, Russia

\section{Valery V. Tuchin, PhD, DSc}

Research-Educational Institute of Optics and Biophotonics, Saratov National Research State University, Institute of Precision Mechanics and Control of the RAS, Saratov, Russia

Samara National Research University, Samara, Russia 